\title{
Post-Mastectomy Radiotherapy After Neoadjuvant Chemotherapy in Breast Cancer: A Pooled Retrospective Analysis of Three Prospective Randomized Trials
}

\author{
David Krug, $\mathrm{MD}^{1,2,21}$, Bianca Lederer, $\mathrm{PhD}^{3}$, Fenja Seither, $\mathrm{MSc}^{3}$, Valentina Nekljudova, $\mathrm{PhD}^{3}$, \\ Beyhan Ataseven, $\mathrm{MD}^{4}$, Jens-Uwe Blohmer, $\mathrm{MD}^{5}$, Serban Dan Costa, $\mathrm{MD}^{6}$, Carsten Denkert, $\mathrm{MD}^{7}$, \\ Nina Ditsch, $\mathrm{MD}^{8}$, Bernd Gerber, $\mathrm{MD}^{9}$, Claus Hanusch, $\mathrm{MD}^{10}$, Joerg Heil, $\mathrm{MD}^{11}$, Jörn Hilfrich, $\mathrm{MD}^{12}$, \\ Jens B. Huober, $\mathrm{MD}^{13}$, Christian Jackisch, $\mathrm{MD}^{14}$, Sherko Kümmel, $\mathrm{MD}^{15}$, Stefan Paepke, $\mathrm{MD}^{16}$, \\ Christian Schem, $\mathrm{MD}^{17}$, Andreas Schneeweiss, $\mathrm{MD}^{18}$, Michael Untch, $\mathrm{MD}^{19}$, Jürgen Debus, MD, PhD ${ }^{1,2}$, \\ Gunter von Minckwitz, $\mathrm{MD}^{3}$, Thorsten Kühn, $\mathrm{MD}^{20}$, and Sibylle Loibl, $\mathrm{MD}^{3}$ \\ ${ }^{1}$ Department of Radiation Oncology, University Hospital Heidelberg, Heidelberg, Germany; ${ }^{2}$ National Center for \\ Radiation Oncology (NCRO), Heidelberg Institute for Radiation Oncology (HIRO), Heidelberg, Germany; ${ }^{3}$ German Breast \\ Group, Neu-Isenburg, Germany; ${ }^{4}$ Department of Gynecology and Gynecologic Oncology, Kliniken Essen-Mitte, Essen, \\ Germany; ${ }^{5}$ Charité, Klinik für Gynäkologie, Berlin, Germany; ${ }^{6}$ Universitätsklinikum Magdeburg, Universitätsfrauenklinik, \\ Magdeburg, Germany; ${ }^{7}$ Institute for Pathology, Philipps-University Marburg, Marburg, Germany; ${ }^{8}$ Department of \\ Obstetrics and Gynecology, Ludwig-Maximilians-University of Munich, Munich, Germany; ${ }^{9}$ Department of Gynecology \\ and Obstetrics, University Hospital, Rostock, Germany; ${ }^{10}$ Rotkreuzklinikum München, Frauenklinik, Munich, Germany; \\ ${ }^{11}$ Breast Unit, University Hospital, University of Heidelberg, Heidelberg, Germany; ${ }^{12}$ Frauenklinik Henriettenstiftung, \\ Hannover, Germany; ${ }^{13}$ Universitätsklinikum Ulm, Universitätsfrauenklinik, Ulm, Germany; ${ }^{14}$ Sana Klinikum Offenbach, \\ Klinik für Gynäkologie und Geburtshilfe, Offenbach, Germany; ${ }^{15}$ Breast Unit, Kliniken Essen-Mitte, Essen, Germany; \\ ${ }^{16}$ Klinikum rechts der Isar TU München, Frauenklinik und Poliklinik, Munich, Germany; ${ }^{17}$ Mammazentrum Hamburg, \\ Hamburg, Germany; ${ }^{18}$ National Center for Tumor Diseases, Heidelberg, Germany; ${ }^{19}$ Helios Klinikum Berlin-Buch, Klinik \\ für Gynäkologie und Geburtshilfe, Berlin, Germany; ${ }^{20}$ Department for Gynecology and Obstetrics, Interdisciplinary Breast \\ Center, Esslingen, Germany; ${ }^{21}$ Present Address: Department of Radiation Oncology, University Hospital Schleswig- \\ Holstein, Kiel, Germany
}

\section{ABSTRACT}

Background. The impact of locoregional radiotherapy (RT) after neoadjuvant chemotherapy (NACT) and mastectomy in breast cancer patients is currently unclear. Several publications have suggested that patients with a

D. Krug, MD

e-mail: david.krug@uksh.de favorable response to NACT might not benefit from RT after mastectomy.

Methods. A retrospective analysis of three prospective randomized NACT trials was performed. Information on the use of RT was available for 817 breast cancer patients with non-inflammatory breast cancer who underwent mastectomy after NACT within the GeparTrio, GeparQuattro, and GeparQuinto-trials. RT was administered to 676 of these patients $(82.7 \%)$.

Results. The 5-year cumulative incidence of locoregional recurrence (LRR) was $15.2 \%$ (95\% confidence interval [CI] 9.0-22.8\%) in patients treated without RT and $11.3 \%$ in patients treated with RT (95\% CI 8.7-14.3\%). In the multivariate analysis, RT was associated with a lower risk of LRR (hazard ratio $0.51,95 \%$ CI $0.27-1.0 ; p=0.05$ ). This effect was shown especially in patients with cT3/4 tumors, as well as in patients who were $\mathrm{cN}+$ before 
neoadjuvant therapy, including those who converted to ypN0 after neoadjuvant therapy. In the bivariate analysis, disease-free survival was significantly worse in patients who received RT, however this was not confirmed in the multivariate analysis.

Conclusions. Our results suggest that RT reduces the LRR rates in breast cancer patients who receive a mastectomy after NACT without an improvement in DFS. Prospective randomized controlled trials such as the National Surgical Adjuvant Breast and Bowel Project B-51/RTOG 1304 trial will analyze whether RT has any benefit in patients who have a favorable response after NACT.

Neoadjuvant chemotherapy (NACT) for breast cancer has enjoyed increasing popularity during the last decade. ${ }^{1}$ The outcome after NACT is at least equivalent to postoperative administration in terms of survival., ${ }^{2,3}$ Treatment response to NACT, especially pathological complete response (pCR) is a strong prognostic factor in individual patients, especially those with triple-negative or human epidermal growth factor receptor 2 (HER2)-overexpressing tumors. ${ }^{4,5}$

There is a clear effect of systemic therapy on the risk of locoregional recurrence (LRR). ${ }^{6}$ Although the absolute benefit of adjuvant post-mastectomy radiotherapy (PMRT) on LRR likely decreases when an effective systemic therapy regimen is administered, its effect on survival is thought to be largest in this context. ${ }^{7,8}$ The question remains as to what extent PMRT improves locoregional control and survival with contemporary systemic therapy since the trials included in the Early Breast Cancer Trialists' Collaborative Group (EBCTCG) meta-analysis on PMRT used outdated systemic therapy regimens. ${ }^{9,10}$ Furthermore, we need additional evidence as to whether
PMRT improves results in patients with response to NACT, especially with a pCR or conversion from clinically involved lymph nodes $(\mathrm{cN}+)$ to pathologically negative lymph nodes (ypNO). ${ }^{11,12}$

We pooled information on the use of PMRT from three prospective randomized controlled trials ${ }^{13-19}$ investigating different NACT regimens, to analyze the impact of adjuvant PMRT on LRR and survival.

\section{PATIENTS AND METHODS}

A pooled analysis of three prospective randomized controlled trials testing different regimens of NACT in breast cancer patients was performed; the results of these trials (GeparTrio, GeparQuattro, and GeparQuinto) have been published elsewhere. ${ }^{13-19}$

A total of 6139 patients were treated in these trials from September 2002 to July 2010. Figure 1 shows the Consolidated Standards of Reporting Trials (CONSORT) diagram for patients included in this analysis. We excluded patients with progression or death before surgery, patients with missing surgery data, patients without further randomization after two initial cycles of TAC (docetaxel, doxorubicin, and cyclophosphamide) in GeparTrio, patients with inflammatory breast cancer, and those who did not receive radiotherapy (RT) due to early progression before surgery. Information on the administration of RT was collected prospectively. Clinical data and follow-up were available from 817 of 1569 patients who had a mastectomy. Details on treatment regarding systemic therapy in GeparTrio, GeparQuattro, and GeparQuinto are available in "Electronic supplementary material".

The study protocols for all three trials provided recommendations regarding the use of RT after NACT and surgery (electronic supplementary Table 1). The choice

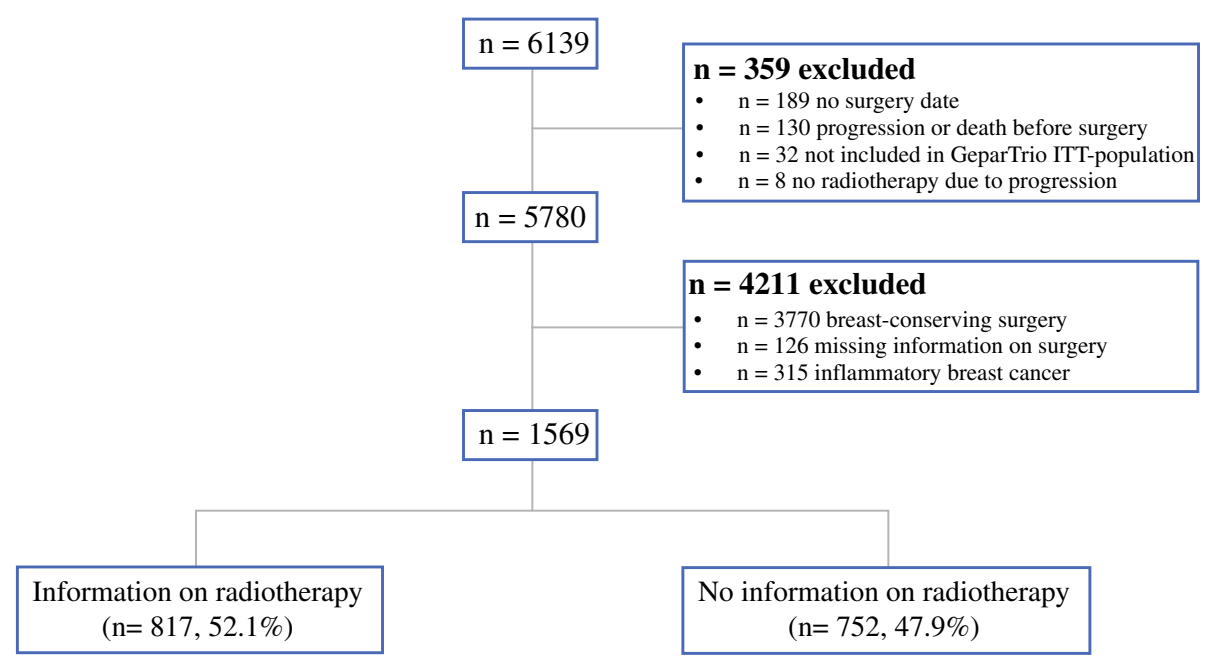

FIG. 1 Overview of patients included in the current analysis. ITT intention to treat 
TABLE 1 Patient characteristics

\begin{tabular}{|c|c|c|c|c|}
\hline Parameter & $\begin{array}{l}\text { All patients } \\
(n=817)\end{array}$ & $\begin{array}{l}\mathrm{RT} \\
(n=676)\end{array}$ & $\begin{array}{l}\text { No RT } \\
(n=141)\end{array}$ & $\begin{array}{l}p \text { value } \\
\text { (RT vs. no RT) }\end{array}$ \\
\hline Age, years [median $(\min -\max )]$ & $49(21-78)$ & $49(21-78)$ & $48(23-68)$ & \\
\hline Age group (years) & & & & 0.004 \\
\hline$<40$ & $123(15.1)$ & $89(13.2)$ & $34(24.1)$ & \\
\hline $40-49$ & $303(37.1)$ & $258(38.2)$ & $45(31.9)$ & \\
\hline$\geq 50$ & $391(47.9)$ & $329(48.7)$ & $62(44.0)$ & \\
\hline Histology & & & & 0.371 \\
\hline Ductal invasive & $623(76.3)$ & $511(75.6)$ & $112(79.4)$ & \\
\hline Lobular invasive & $145(17.7)$ & $121(17.9)$ & $24(17.0)$ & \\
\hline Other & $49(6.0)$ & $44(6.5)$ & $5(3.5)$ & \\
\hline Grading & & & & 0.115 \\
\hline G1 & $31(3.8)$ & $30(4.4)$ & $1(0.7)$ & \\
\hline G2 & $483(59.1)$ & $398(58.9)$ & $85(60.3)$ & \\
\hline G3 & $271(33.2)$ & $224(33.1)$ & $47(33.3)$ & \\
\hline Missing & $32(3.9)$ & $24(3.6)$ & $8(5.7)$ & \\
\hline ER status & & & & 0.921 \\
\hline ER-positive & $556(68.1)$ & $459(67.9)$ & $97(68.8)$ & \\
\hline ER-negative & $258(31.6)$ & $214(31.7)$ & $44(31.2)$ & \\
\hline Missing & $3(0.4)$ & $3(0.4)$ & 0 & \\
\hline PR status & & & & 0.926 \\
\hline PR-positive & $464(56.8)$ & $384(56.8)$ & $80(56.7)$ & \\
\hline PR-negative & $349(42.7)$ & $288(42.6)$ & $61(43.3)$ & \\
\hline Missing & $4(0.5)$ & $4(0.6)$ & 0 & \\
\hline HER2 status & & & & 0.746 \\
\hline HER2-positive & $207(25.3)$ & $170(25.1)$ & $37(26.2)$ & \\
\hline HER2-negative & $564(69.0)$ & $470(69.5)$ & $94(66.7)$ & \\
\hline Missing & $46(5.6)$ & $36(5.3)$ & $10(7.1)$ & \\
\hline Breast cancer subtype & & & & 0.816 \\
\hline HR+/HER2-/G1-2 & $323(39.5)$ & $272(40.2)$ & $51(36.2)$ & \\
\hline HR+/HER2-/G3 & $103(12.6)$ & $87(12.9)$ & $16(11.3)$ & \\
\hline $\mathrm{HR}+/ \mathrm{HER} 2+$ & $116(14.2)$ & $93(13.8)$ & $23(16.3)$ & \\
\hline HR-/HER2+ & $90(11.0)$ & $76(11.2)$ & $14(9.9)$ & \\
\hline $\mathrm{TNBC}$ & $128(15.7)$ & $104(15.4)$ & $24(17.0)$ & \\
\hline Missing & $57(7.0)$ & $44(6.5)$ & $13(9.2)$ & \\
\hline Clinical tumor stage & & & & $<0.001$ \\
\hline $\mathrm{cT} 1$ & $38(4.7)$ & $28(4.1)$ & $10(7.1)$ & \\
\hline $\mathrm{cT} 2$ & $403(49.3)$ & $315(46.6)$ & $88(62.4)$ & \\
\hline cT3 & $253(31.0)$ & $222(32.8)$ & $31(22.0)$ & \\
\hline $\mathrm{cT} 4 \mathrm{a}-\mathrm{c}$ & 119 (14.6) & $108(16.0)$ & $11(7.8)$ & \\
\hline Missing & $4(0.5)$ & $3(0.4)$ & $1(0.7)$ & \\
\hline Clinical nodal stage & & & & 0.001 \\
\hline cNO & $309(37.8)$ & $238(35.2)$ & $71(50.4)$ & \\
\hline $\mathrm{cN}+$ & $501(61.3)$ & $432(63.9)$ & $69(48.9)$ & \\
\hline Missing & $7(0.9)$ & $6(0.9)$ & $1(0.7)$ & \\
\hline Initial SLNB & & & & 0.484 \\
\hline Yes & $74(9.1)$ & $61(9.0)$ & $13(9.2)$ & \\
\hline No & $448(54.8)$ & $383(56.7)$ & $65(46.1)$ & \\
\hline Missing & $295(36.1)$ & $232(34.3)$ & $63(44.7)$ & \\
\hline
\end{tabular}


TABLE 1 continued

\begin{tabular}{|c|c|c|c|c|}
\hline Parameter & $\begin{array}{l}\text { All patients } \\
(n=817)\end{array}$ & $\begin{array}{l}\mathrm{RT} \\
(n=676)\end{array}$ & $\begin{array}{l}\text { No RT } \\
(n=141)\end{array}$ & $\begin{array}{l}p \text { value } \\
\text { (RT vs. no RT) }\end{array}$ \\
\hline SLNB-positive (valid \%) & $43(61.4)$ & $40(69.0)$ & $3(25.0)$ & $<0.001$ \\
\hline Pathological tumor stage & & & & 0.001 \\
\hline урт0 & $78(9.5)$ & $58(8.6)$ & $20(14.2)$ & \\
\hline урТ1 & $207(25.3)$ & $158(23.4)$ & $49(34.8)$ & \\
\hline урт2 & $173(21.2)$ & $151(22.3)$ & $22(15.6)$ & \\
\hline урT3 & $115(14.1)$ & $109(16.1)$ & $6(4.3)$ & \\
\hline урT4a-c & $25(3.1)$ & $24(3.6)$ & $1(0.7)$ & \\
\hline ypT4d & $5(0.6)$ & $5(0.7)$ & 0 & \\
\hline$>$ ypT0 & $172(21.1)$ & $139(20.7)$ & $33(23.4)$ & \\
\hline ypTis & $37(4.5)$ & $28(4.2)$ & $9(6.4)$ & \\
\hline Missing & $5(0.6)$ & $4(0.6)$ & $1(0.7)$ & \\
\hline Pathological nodal stage & & & & $<0.001$ \\
\hline ypN0 & $335(41.0)$ & $241(35.7)$ & $94(66.7)$ & \\
\hline ypN+ & $464(56.8)$ & $421(62.3)$ & $43(30.5)$ & \\
\hline Missing & $18(2.2)$ & $14(2.1)$ & $4(2.8)$ & \\
\hline \multicolumn{5}{|l|}{ Axillary surgical procedure } \\
\hline Axillary dissection & $694(84.9)$ & $586(86.7)$ & $108(76.6)$ & $<0.001$ \\
\hline SLNB alone & $26(3.2)$ & $14(2.1)$ & $12(8.5)$ & \\
\hline None & $13(1.6)$ & $9(1.3)$ & $4(2.8)$ & \\
\hline Missing & $84(10.3)$ & $67(9.9)$ & $17(12.1)$ & \\
\hline Median number of removed lymph nodes (IQR) & $15(11-19)$ & $15(12-20)$ & $13(10-18)$ & \\
\hline урT0 урN0 & & & & 0.089 \\
\hline Yes & $67(8.2)$ & $50(7.4)$ & $17(12.1)$ & \\
\hline No & $750(91.8)$ & $626(92.6)$ & $124(87.9)$ & \\
\hline урT0/Tis ypN0 & & & & 0.020 \\
\hline Yes & 95 (11.6) & $70(10.4)$ & $25(17.7)$ & \\
\hline No & $722(88.4)$ & $606(89.6)$ & $116(82.3)$ & \\
\hline
\end{tabular}

Data are expressed as $n(\%)$ unless otherwise specified

$R T$ radiotherapy, $S L N B$ sentinel lymph node biopsy, $I Q R$ interquartile range, min minimum, max maximum, $E R$ estrogen receptor, $P R$ progesterone receptor, HER2 human epidermal growth factor receptor, $H R$ hormone receptor, TNBC triple-negative breast cancer

between breast-conserving surgery and mastectomy after NACT was left to the discretion of the local investigator. Surgery was scheduled 1-14 days after completion of the last cycle of chemotherapy or at least 28 days after the last infusion of bevacizumab for patients in the GeparQuinto trial.

Endocrine therapy for a duration of at least 5 years was recommended to all patients with estrogen and/or progesterone receptor-positive breast cancer. One year of trastuzumab was administered to patients with HER2overexpressing tumors in the GeparQuattro and GeparQuinto trials, while no trastuzumab was administered to patients in the GeparTrio trial.

Information on the use of RT was prospectively collected from the case report forms (CRFs), however the impact of RT was not a predefined endpoint in any of the included trials. The CRF design differed between the three trials and only contained limited information regarding target volume, RT dose, and fractionation. Thus, the original RT reports were requested from the study centers and reviewed by the first author.

\section{Endpoints and Covariates}

All endpoints and covariates were assessed by local investigators, while histopathological reports were assessed by an experienced pathologist. Follow-up was performed according to local guidelines and was monitored at least every 6 months for 5 years after diagnosis.

pCR was defined as the absence of both invasive and non-invasive residual tumor in the breast and ipsilateral axillary lymph nodes (ypT0 ypN0). Patients were grouped according to their clinical lymph node status at first diagnosis $(\mathrm{cN}+$ vs. $\mathrm{cN} 0)$ and their pathological lymph node 
status after NACT (ypN+ vs. ypN0). LRR included chest wall relapses and regional lymph node metastases, and was calculated from the date of surgery until the occurrence of an LRR. Only LRR events occurring before or simultaneously with distant recurrence were evaluated. Disease-free survival (DFS) was defined as the time from surgery until the occurrence of a locoregional or distant recurrence, or death from any cause.

All three trials were approved by the local Ethics Committees, and written informed consent was obtained from all patients before registration.

\section{Statistics}

Statistical analysis was performed using SPSS software version 22.0 (IBM Corporation, Armonk, NY, USA) and SAS version 9.4 (SAS Institute, Cary, NC, USA). Baseline patient characteristics were compared using the two-sided Pearson Chi square test, and Cox regression analysis was used for univariate and multivariate analyses regarding DFS.

The cumulative incidence functions of LRR using distant recurrence as the competing event were compared using Gray's test, with alpha $=0.05$ stratified for study. Bivariate analyses adjusted for the trial were used for LRR and DFS.

Multivariate competing risk analysis of LRR using distant recurrence as the competing event was performed using the Fine-Gray model. Hazard ratios (HRs) and 95\% confidence intervals (CIs) are given.

Multivariate analysis was adjusted for known prognostic factors, i.e. clinical tumor and lymph node stage, age, hormone receptor and HER2 receptor status, histologic subtype, grading, response to chemotherapy, and trial. A two-sided $p$ value of 0.05 was used as the alpha error for consideration of statistical significance.

\section{RESULTS}

The CONSORT diagram according to the use of RT is depicted in Fig. 1. Median follow-up was 51.5 months from surgery. Of the 817 patients included in this analysis, $676(82.7 \%)$ received adjuvant $\mathrm{RT}$ to the chest wall and/or the regional lymph nodes (Fig. 1). Baseline and posttherapeutic patient characteristics for patients with information on RT are listed in Table 1. Detailed reports were available from 318 patients (46.4\%) treated with RT; 313 of these patients $(98.7 \%)$ received RT to the thoracic wall. RT to the regional lymph nodes was delivered to 243 of 318 patients (76.7\%) and included the supraclavicular/infraclavicular lymph nodes in 237 of 318 patients (74.5\%), the parasternal (internal mammary) lymph nodes in 49 patients $(15.4 \%)$, and the axillary lymph nodes in 58 patients $(18.2 \%)$. Seventy-two patients $(22.6 \%)$ were treated with an additional boost irradiation to the mastectomy scar. Electronic supplementary Table 2 shows the patient and tumor characteristics for the 817 patients with available CRF information regarding RT, and the 752 patients without information on RT.

Patients who did not receive RT were significantly younger than patients who received RT. In addition, patients who received RT had significantly more advanced tumor and nodal stages before and after NACT and were more likely to have an axillary dissection. There was a significantly lower rate of $\mathrm{pCR}$ in the group who received RT when non-invasive residual disease in the breast was also considered (ypT0/is ypN0, $17.7 \%$ with RT vs. $10.4 \%$ without $\mathrm{RT} ; p=0.02)$.

\section{Locoregional Recurrence}

The 5-year cumulative incidence of LRR was $15.2 \%$ (95\% CI 9.0-22.8\%) in patients treated without RT and $11.3 \%$ in patients treated with adjuvant RT $(95 \%$ CI 8.7-14.3). The cumulative incidence functions are shown in Fig. 2. Using multivariate competing risk analysis, there was no statistically significant difference between patients who had or had not received adjuvant RT $(p=0.23)$. Table 2 lists the 5-year cumulative incidence of LRR and the results of the bivariate competing risk analysis. LRR in patients with cT3/4 tumors was significantly lower after locoregional RT. There was a borderline significant result for patients with $\mathrm{cN}+$ and patients who converted from $\mathrm{cN}+$ to ypN0, and a trend towards lower LRR in patients with ypNO. In patients who had a pCR after NACT, there was no significant difference whether locoregional RT was used or not.

In the multivariate analysis, estrogen receptor, progesterone receptor, and baseline nodal status were significant prognostic factors for LRR, while the use of adjuvant RT was of borderline significance, with a $p$ value of 0.05 (Table 3).

We carried out multivariate analyses according to baseline tumor and nodal stage, pCR, and nodal stage after NACT. No significant effect of RT was found in patients who did or did not achieve a pCR. Adjuvant RT did not result in improved outcomes for patients with cT1/2 tumors, but did improve results for patients with cT3/4 tumors (HR 0.4, 95\% CI 0.17-0.94; $p=0.04$ ). Similar results were found according to baseline nodal status, where patients with $\mathrm{cN}+$ had a significantly better outcome when treated with RT (HR 0.39, 95\% CI 0.19-0.83; $p=0.01$ ), whereas patients with cN0 showed similar results with or without RT. Patients with ypN0 did significantly benefit from RT (HR 0.2 , 95\% CI 0.06-0.62; 
TABLE 2 Cumulative incidence of locoregional recurrence and bivariate competing risk analysis adjusted for trial in relation to pCR, clinical lymph node status before neoadjuvant chemotherapy $(\mathrm{cN}+/ \mathrm{cN} 0)$, and pathological lymph node status after neoadjuvant chemotherapy $(\mathrm{ypN}+/$ ypN0)

\begin{tabular}{|c|c|c|c|c|}
\hline \multirow[t]{3}{*}{ Parameter } & \multicolumn{4}{|c|}{ 5-Year locoregional recurrence rate } \\
\hline & $\begin{array}{l}\text { Radiotherapy } \\
\text { (95\% CI) }\end{array}$ & $\begin{array}{l}\text { No radiotherapy } \\
(95 \% \mathrm{CI})\end{array}$ & HR $(95 \% \mathrm{CI})$ & $p$ value \\
\hline & {$[n=676]$} & {$[n=141]$} & & \\
\hline \multirow{3}{*}{$\begin{array}{l}\mathrm{pCR} \\
{[n=67]}\end{array}$} & $5.8 \%$ & $13.8 \%$ & $0.2(0.02-2.39)$ & 0.21 \\
\hline & $(0.3-24.0 \%)$ & $(2.0-36.6 \%)$ & & \\
\hline & {$[n=50]$} & {$[n=17]$} & & \\
\hline \multirow{3}{*}{$\begin{array}{l}\text { No pCR } \\
{[n=750]}\end{array}$} & $11.8 \%$ & $15.4 \%$ & $0.74(0.42-1.30)$ & 0.3 \\
\hline & $(9.1-15.0 \%)$ & $(8.8-23.7 \%)$ & & \\
\hline & {$[n=626]$} & {$[n=124]$} & & \\
\hline \multirow{3}{*}{$\begin{array}{l}\mathrm{cT} 1 / 2 \\
{[n=441]}\end{array}$} & $10.3 \%$ & $10.5 \%$ & $0.94(0.45-1.95)$ & 0.86 \\
\hline & $(7.2-14.0 \%)$ & $(4.8-18.8 \%)$ & & \\
\hline & {$[n=343]$} & {$[n=98]$} & & \\
\hline \multirow{3}{*}{$\begin{array}{l}\mathrm{cT} 3 / 4 \\
{[n=372]}\end{array}$} & $12.2 \%$ & $24.9 \%$ & $0.43(0.19-0.95)$ & 0.04 \\
\hline & $(8.3-16.9 \%)$ & $(11.8-40.6 \%)$ & & \\
\hline & {$[n=330]$} & {$[n=42]$} & & \\
\hline \multirow{3}{*}{$\begin{array}{l}\text { cN0 } \\
{[n=309]}\end{array}$} & $8.1 \%$ & $8.9 \%$ & $0.89(0.28-2.83)$ & 0.84 \\
\hline & $(4.3-13.4 \%)$ & $(3.2-18.4 \%)$ & & \\
\hline & {$[n=238]$} & {$[n=71]$} & & \\
\hline \multirow{3}{*}{$\begin{array}{l}\mathrm{cN}+ \\
{[n=501]}\end{array}$} & $13.3 \%$ & $22.9 \%$ & $0.54(0.29-0.99)$ & 0.05 \\
\hline & $(9.9-17.2 \%)$ & $(11.6-36.5 \%)$ & & \\
\hline & {$[n=432]$} & {$[n=69]$} & & \\
\hline \multirow{3}{*}{$\begin{array}{l}\text { ypN0 } \\
{[n=335]}\end{array}$} & $7.5 \%$ & $13.7 \%$ & $0.44(0.19-1.02)$ & 0.06 \\
\hline & $(4.0-12.4 \%)$ & $(6.8-23.1 \%)$ & & \\
\hline & {$[n=241]$} & {$[n=94]$} & & \\
\hline \multirow{3}{*}{$\begin{array}{l}\text { ypN+ } \\
{[n=464]}\end{array}$} & $13.6 \%$ & $17.0 \%$ & $0.81(0.34-1.95)$ & 0.64 \\
\hline & $(10.1-17.6 \%)$ & $(6.7-31.2 \%)$ & & \\
\hline & {$[n=421]$} & {$[n=43]$} & & \\
\hline \multirow{3}{*}{$\begin{array}{l}\mathrm{cN}+\rightarrow \text { ypN0 } \\
{[n=158]}\end{array}$} & $9.3 \%$ & $22.2 \%$ & $0.37(0.14-0.99)$ & 0.05 \\
\hline & $(4.4-16.4 \%)$ & $(7.9-40.9 \%)$ & & \\
\hline & {$[n=121]$} & {$[n=37]$} & & \\
\hline
\end{tabular}

$p C R$ pathological complete response, $H R$ hazard ratio, $C I$ confidence interval

$p=0.01)$, while patients with $\mathrm{ypN}+$ did not. In the subgroup of patients who converted from $\mathrm{cN}+$ to ypN0, RT was associated with a lower risk of LRR (HR 0.19, 95\% CI 0.04-0.97; $p=0.05$ ).

\section{Disease-Free Survival}

Overall, DFS was significantly worse for patients who received RT compared with patients who did not receive $\mathrm{RT}$ in the bivariate analysis (HR 1.48, 95\% CI 1.01-2.18; $p=0.04)$. However, RT was not associated with an inferior DFS upon multivariate analysis (HR 1.14, 95\% CI $0.75-1.73 ; p=0.55$ ), as shown in Table 3. Patients who were clinically node negative at diagnosis and received RT had a significantly worse DFS upon multivariate analysis
(HR 3.4, 95\% CI 1.46-7.91; $p=0.01$ ). In all other multivariate subgroup analyses, RT was not significantly associated with DFS.

\section{DISCUSSION}

Our results from this retrospective explorative analysis of three large prospective randomized phase III trials suggest that RT decreases the risk of LRR in breast cancer patients who have a mastectomy after NACT without an improvement in DFS.

The positive impact of RT on LRR and survival has been shown in large meta-analyses, both after breast-conserving surgery and after mastectomy in the adjuvant setting. ${ }^{20}$ However, most of the randomized trials included 


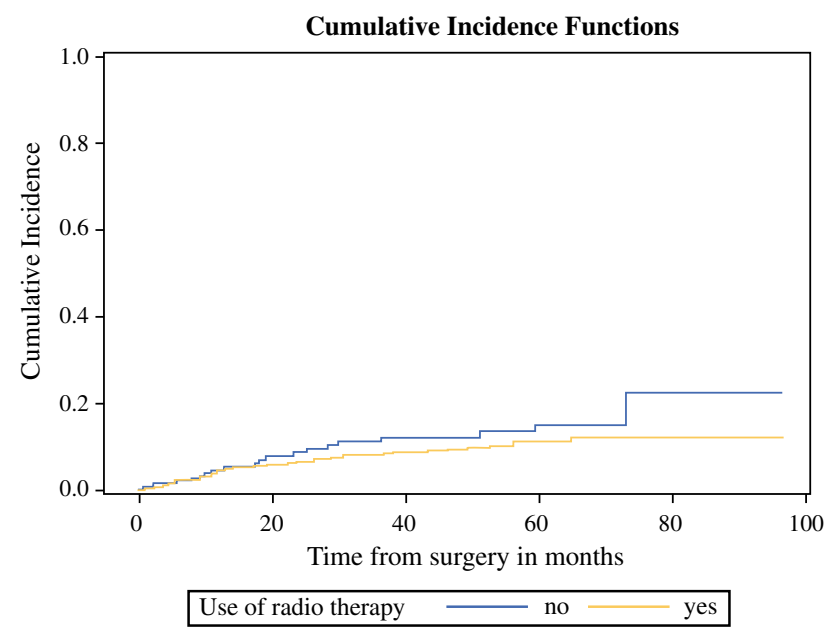

FIG. 2 Kaplan-Meier plot for cumulative incidence of locoregional recurrence

in these meta-analyses used adjuvant systemic therapy regimens considered suboptimal according to contemporary standards.

While two meta-analyses based on published data found no increased risk of LRR with NACT compared with adjuvant chemotherapy when trials with suboptimal local treatment were excluded, ${ }^{2,3}$ the recently published EBCTCG meta-analysis based on individual patient data showed an increase in local recurrence, even in this context. $^{21}$

Several studies showed that a favorable treatment response, e.g. pCR or ypN0, correlates with a decreased risk of locoregional failure, while the overall risk of LRR is influenced by both pre- and post-therapeutic tumor and nodal stage. ${ }^{9,10}$

Evidence on the role of RT after NACT followed by mastectomy is limited. A systematic review on post-mastectomy RT identified 24 publications, of which 23 were single-center, retrospective reports. ${ }^{22}$ While some of those studies implied that the omission of $\mathrm{RT}$ is possible in selected patients after NACT and mastectomy without compromising locoregional control or survival, ${ }^{23-25}$ other researchers have shown that RT improved both endpoints, even in subgroups of patients with a $\mathrm{pCR} .{ }^{26} \mathrm{~A}$ recent systematic review of publications studying the possibility of RT treatment individualization based on treatment response yielded 13 retrospective studies, mostly from single institutions. ${ }^{27}$

In our analysis, the incidence of LRR was higher than in the NSABP B18/27 analysis and the I-SPY 1 trial. ${ }^{25,28}$ This is most likely related to negative patient selection in the present analysis, with $>45 \%$ of patients presenting with cT3/4 disease and $>60 \%$ presenting with clinically involved lymph nodes. Although the great majority of patients received RT and the absolute numbers of recurrences were low, we were able to demonstrate that RT is associated with a decreased risk of LRR without a DFS benefit. The most intriguing finding is that patients with negative axillary lymph nodes at surgery (ypNO) had a significantly lower risk of LRR when treated with RT (HR $0.2,95 \%$ CI $0.06-0.62 ; p=0.01)$. However, it should be mentioned that $61.3 \%$ of included patients had clinically involved lymph nodes and $45.6 \%$ had cT3-4 tumors. Both subgroups had a significantly lower risk of LRR when treated with RT in the absence of a DFS benefit, while patients with $\mathrm{cN} 0$ had no benefit from RT and showed a worse DFS (HR 3.4, 95\% CI 1.46-7.91; $p=0.01$ ). Interestingly, age was not associated with LRR on multivariate analysis, which is line with findings from the NSABP B18/ 27 analysis. $^{25}$

Other groups have addressed the question as to whether patients with ypNO would benefit from RT after mastectomy. Two retrospective studies showed no benefit of RT on LRR or survival, however these studies included a substantial amount of cNO patients. ${ }^{24,29}$ Several other publications suggest that the benefit from RT is highly dependent on the pretherapeutic tumor and nodal stage, and that selected subgroups with advanced disease can derive a significant advantage both in terms of LRR and survival. $^{23,26,30}$

The finding that patients with $\mathrm{cNO}$ had a significantly worse DFS on multivariate analysis might be related to the presence of a negative selection bias and is not supported by the current evidence. ${ }^{27}$

This study has several limitations. Due to the non-randomized retrospective comparison and the complex interaction between clinical and pathological parameters and the response to NACT, the presence of underlying confounding factors cannot be excluded. Since only patients after mastectomy were included, our study population mostly contained patients with high-risk features such as advanced tumor stage at diagnosis and unfavorable treatment response, which is illustrated by the relatively low pCR rate and high number of patients with pathologically involved lymph nodes after NACT. The treatment regimens were heterogeneous among the three trials and included a response-adapted treatment schedule without anti-HER2 medication in GeparTrio, and a second randomization for non-responders in GeparQuinto. In addition, the RT recommendations differed between GeparTrio and GeparQuattro/GeparQuinto. A detailed analysis of target volume, radiation dose, and fractionation, all of which might impact patient outcome, was not possible due to missing information.

Since the included trials were conducted before publication of several prospective trials supporting its use, ${ }^{31,32}$ irradiation of the internal mammary lymph nodes was 
TABLE 3 Results of the multivariate Cox regression analysis for locoregional recurrence and disease-free survival

\begin{tabular}{|c|c|c|c|c|}
\hline Parameter & $\begin{array}{l}\text { LRR } \\
{[\text { HR }(95 \% \text { CI })]}\end{array}$ & $\begin{array}{l}\text { LRR } \\
p \text { value }\end{array}$ & $\begin{array}{l}\text { DFS } \\
{[\mathrm{HR}(95 \% \mathrm{CI})]}\end{array}$ & $\begin{array}{l}\text { DFS } \\
p \text { value }\end{array}$ \\
\hline Age (years) & & 0.34 & & 0.42 \\
\hline$\geq 50$ & 1.0 (Ref) & & 1.0 (Ref) & \\
\hline $40-49$ & $0.7(0.4-1.21)$ & 0.2 & $1.22(0.90-1.67)$ & 0.21 \\
\hline$<40$ & $1.08(0.54-2.15)$ & 0.82 & $1.19(0.79-1.80)$ & 0.41 \\
\hline Histology & & 0.31 & & 0.39 \\
\hline Ductal invasive & 1.0 (Ref) & & 1.0 (Ref) & \\
\hline Lobular invasive & $0.64(0.28-1.43)$ & 0.28 & $0.73(0.47-1.15)$ & 0.18 \\
\hline Other & $0.42(0.09-1.88)$ & 0.26 & $0.88(0.49-1.61)$ & 0.68 \\
\hline Grading & & 0.51 & & 0.13 \\
\hline G1 & 1.0 (Ref) & & 1.0 (Ref) & \\
\hline $\mathrm{G} 2$ & $0.93(0.23-3.79)$ & 0.91 & $1.80(0.66-4.93)$ & 0.25 \\
\hline G3 & $1.25(0.3-5.17)$ & 0.76 & $2.27(0.82-6.28)$ & 0.11 \\
\hline ER-negative & $4.5(2.42-8.37)$ & $<0.01$ & $1.93(1.33-2.80)$ & $<0.01$ \\
\hline PR-negative & $0.52(0.29-0.96)$ & 0.04 & $1.45(1.01-2.08)$ & 0.05 \\
\hline HER2/neu-positive & $0.58(0.32-1.06)$ & 0.08 & $0.66(0.47-0.93)$ & 0.02 \\
\hline $\mathrm{cT}$ & & 0.71 & & $<0.01$ \\
\hline $\mathrm{cT} 1$ & 1.0 (Ref) & & 1.0 (Ref) & \\
\hline $\mathrm{cT} 2$ & $1.59(0.39-6.57)$ & 0.52 & $3.07(0.96-9.84)$ & 0.06 \\
\hline cT3 & $1.45(0.35-6.04)$ & 0.61 & $4.04(1.26-13.00)$ & 0.02 \\
\hline $\mathrm{cT} 4 \mathrm{a}-\mathrm{c}$ & $2.13(0.45-10.11)$ & 0.34 & $5.98(1.81-19.75)$ & $<0.01$ \\
\hline $\mathrm{cN}+$ & $2.14(1.19-3.87)$ & 0.01 & $1.87(1.35-2.60)$ & $<0.01$ \\
\hline Study number & & 0.53 & & 0.64 \\
\hline GeparQuinto & 1.0 (Ref) & & 1.0 (Ref) & \\
\hline GeparTrio & $0.78(0.43-1.41)$ & 0.41 & $0.98(0.70-1.37)$ & 0.89 \\
\hline GeparQuattro & $0.7(0.36-1.36)$ & 0.29 & $0.85(0.58-1.23)$ & 0.38 \\
\hline pCR [ypT0 ypN0] & $0.38(0.11-1.29)$ & 0.12 & $0.38(0.2-0.71)$ & $<0.01$ \\
\hline Radiotherapy & $0.51(0.27-1.0)$ & 0.05 & $1.14(0.75-1.73)$ & 0.55 \\
\hline
\end{tabular}

$p C R$ pathological complete response, $H R$ hazard ratio, $C I$ confidence interval, $L R R$ locoregional recurrence, $D F S$ disease-free survival, $E R$ estrogen receptor, $P R$ progesterone receptor, HER 2 human epidermal growth factor receptor

infrequently used. Since irradiation to the internal mammary lymph nodes has also been shown to significantly improve outcome in patients treated with NACT, ${ }^{33,34}$ its infrequent use might have negatively influenced the outcome of patients who received RT in this analysis.

Despite these limitations, the results of this explorative analysis provide insight into the role of RT after NACT followed by mastectomy, and suggest that RT delivered to the chest wall \pm supraclavicular nodes decreases LRR without a benefit in terms of DFS.

\section{CONCLUSIONS}

While individualization of locoregional RT based on treatment response is a concept worth pursuing, results from prospective randomized controlled trials including meticulous quality assurance, such as the NSABP B-51/ RTOG 1304 trial (NCT01872975), are needed to guide clinical practice. In the absence of those data, there is currently insufficient evidence for routine omission of adjuvant RT in patients with cT3/4 tumors or clinically involved lymph nodes. ${ }^{11,27,35}$ In patients with cT1-2 cN+ who become ypN0 or have pCR after NACT, the benefit and risk of RT should be discussed based on the individual recurrence risk for each patient.

ACKNOWLEDGMENT The authors thank all participating centers for providing radiotherapy reports. This work was previously presented in part at the 51st Annual Meeting of the American Society of Clinical Oncology, Chicago, IL, USA, 29 May-2 June 2015.

FUNDING Financial support for the original trials was provided by Amgen, Chugai, GlaxoSmithKline, Roche, and Sanofi-Aventis.

DISCLOSURE David Krug, Bianca Lederer, Fenja Seither, Valentina Nekljudova, Beyhan Ataseven, Jens-Uwe Blohmer, Serban 
Dan Costa, Carsten Denkert, Nina Ditsch, Bernd Gerber, Claus Hanusch, Joerg Heil, Jörn Hilfrich, Jens B. Huober, Christian Jackisch, Sherko Kümmel, Stefan Paepke, Christian Schem, Andreas Schneeweiss, Michael Untch, Jürgen Debus, Gunter von Minckwitz, Thorsten Kühn, and Sibylle Loibl have no conflicts of interest relevant to this study.

\section{REFERENCES}

1. Mougalian SS, Soulos PR, Killelea BK, et al. Use of neoadjuvant chemotherapy for patients with stage I to III breast cancer in the United States. Cancer. 2015;121(15):2544-52.

2. Mauri D, Pavlidis N, Ioannidis JPA. Neoadjuvant versus adjuvant systemic treatment in breast cancer: a meta-analysis. J Natl Cancer Inst. 2005;97(3):188-94.

3. Mieog JSD, van der Hage JA, van de Velde CJH. Neoadjuvant chemotherapy for operable breast cancer. $B r \quad J \quad$ Surg. 2007;94(10):1189-200.

4. Cortazar P, Zhang L, Untch M, et al. Pathological complete response and long-term clinical benefit in breast cancer: the CTNeoBC pooled analysis. Lancet. 2014;384(9938):164-72.

5. von Minckwitz G, Untch M, Blohmer JU, et al. Definition and impact of pathologic complete response on prognosis after neoadjuvant chemotherapy in various intrinsic breast cancer subtypes. J Clin Oncol. 2012;30(15):1796-804.

6. Mamounas EP, Tang G, Liu Q. The importance of systemic therapy in minimizing local recurrence after breast-conserving surgery: the NSABP experience. $J$ Surg Oncol. 2014;110(1):45-50.

7. Poortmans P. Postmastectomy radiation in breast cancer with one to three involved lymph nodes: ending the debate. Lancet. 2014;383(9935):2104-6.

8. Punglia RS, Morrow M, Winer EP, Harris JR. Local therapy and survival in breast cancer. N Engl J Med. 2007;356(23):2399-405.

9. Buchholz TA, Tucker SL, Masullo L, et al. Predictors of localregional recurrence after neoadjuvant chemotherapy and mastectomy without radiation. J Clin. Oncol. 2002;20(1):17-23.

10. Chen AM, Meric-Bernstam F, Hunt KK, et al. Breast conservation after neoadjuvant chemotherapy: the MD Anderson cancer center experience. J Clin Oncol. 2004;22(12):2303-12.

11. Marks LB, Prosnitz LR. Reducing local therapy in patients responding to preoperative systemic therapy: Are we outsmarting ourselves? J Clin Oncol. 2014;32(6):491-3.

12. White J, Mamounas E. Locoregional radiotherapy in patients with breast cancer responding to neoadjuvant chemotherapy: a paradigm for treatment individualization. $J$ Clin Oncol. 2014;32(6):494-5.

13. Huober J, Fasching PA, Hanusch C, et al. Neoadjuvant chemotherapy with paclitaxel and everolimus in breast cancer patients with non-responsive tumours to epirubicin/cyclophosphamide (EC) \pm bevacizumab-results of the randomised GeparQuinto study (GBG 44). Eur J Cancer. 2013;49(10):2284-293.

14. Untch M, Loibl S, Bischoff J, et al. Lapatinib versus trastuzumab in combination with neoadjuvant anthracycline-taxane-based chemotherapy (GeparQuinto, GBG 44): a randomised phase 3 trial. Lancet Oncol. 2012;13(2):135-44.

15. Gerber B, Loibl S, Eidtmann H, et al. Neoadjuvant bevacizumab and anthracycline-taxane-based chemotherapy in 678 triplenegative primary breast cancers; results from the geparquinto study (GBG 44). Ann Oncol. 2013;24(12):2978-84.

16. Untch M, Rezai M, Loibl S, et al. Neoadjuvant treatment with trastuzumab in HER2-positive breast cancer: results from the GeparQuattro study. J Clin Oncol. 2010;28(12):2024-31.
17. von Minckwitz G, Eidtmann H, Rezai M, et al. Neoadjuvant chemotherapy and bevacizumab for HER2-negative breast cancer. N Engl J Med. 2012;366(4):299-309.

18. von Minckwitz G, Blohmer JU, Costa SD, et al. Response-guided neoadjuvant chemotherapy for breast cancer. J Clin Oncol. 2013;31(29):3623-30.

19. von Minckwitz G, Rezai M, Fasching PA, et al. Survival after adding capecitabine and trastuzumab to neoadjuvant anthracycline-taxane-based chemotherapy for primary breast cancer (GBG 40-GeparQuattro). Ann Oncol. 2013;25(1):81-9.

20. EBCTCG (Early Breast Cancer Trialists' Collaborative Group), McGale P, Taylor C, et al. Effect of radiotherapy after mastectomy and axillary surgery on 10-year recurrence and 20-year breast cancer mortality: meta-analysis of individual patient data for 8135 women in 22 randomised trials. Lancet. 2014;383(9935):2127-35.

21. Early Breast Cancer Trialists' Collaborative Group (EBCTCG). Long-term outcomes for neoadjuvant versus adjuvant chemotherapy in early breast cancer: meta-analysis of individual patient data from ten randomised trials. Lancet Oncol. 2018;19(1):27-39.

22. Fowble BL, Einck JP, Kim DN, et al. Role of postmastectomy radiation after neoadjuvant chemotherapy in stage II-III breast cancer. Int J Radiat Oncol Biol Phys. 2012;83(2):494-503.

23. Garg AK, Strom EA, McNeese MD, et al. T3 disease at presentation or pathologic involvement of four or more lymph nodes predict for locoregional recurrence in stage II breast cancer treated with neoadjuvant chemotherapy and mastectomy without radiotherapy. Int J Radiat Oncol Biol Phys. 2004;59(1):138-45.

24. Le Scodan R, Selz J, Stevens D, et al. Radiotherapy for stage II and stage III breast cancer patients with negative lymph nodes after preoperative chemotherapy and mastectomy. Int $J$ Radiat Oncol Biol Phys. 2012;82(1):e1-7.

25. Mamounas EP, Anderson SJ, Dignam JJ, et al. Predictors of locoregional recurrence after neoadjuvant chemotherapy: results from combined analysis of national surgical adjuvant breast and bowel project B-18 and B-27. J Clin Oncol. 2012;30(32):3960-6.

26. McGuire SE, Gonzalez-Angulo AM, Huang EH, et al. Postmastectomy radiation improves the outcome of patients with locally advanced breast cancer who achieve a pathologic complete response to neoadjuvant chemotherapy. Int J Radiat Oncol Biol Phys. 2007;68(4):1004-9.

27. Krug D, Baumann R, Budach W, et al. Individualization of postmastectomy radiotherapy and regional nodal irradiation based on treatment response after neoadjuvant chemotherapy for breast cancer: a systematic review. Strahlenther Onkol. 2018;21:2600-12.

28. Cureton EL, Yau C, Alvarado MD, et al. Local recurrence rates are low in high-risk neoadjuvant breast cancer in the I-SPY 1 Trial (CALGB 150007/150012; ACRIN 6657). Ann Surg Oncol. 2014;21(9):2889-96.

29. Shim SJ, Park W, Huh SJ, et al. The role of postmastectomy radiation therapy after neoadjuvant chemotherapy in clinical stage II-III breast cancer patients with pN0: a multicenter, retrospective study (KROG 12-05). Int J Radiat Oncol Biol Phys. 2014;88(1):65-72.

30. Huang EH, Tucker SL, Strom EA, et al. Postmastectomy radiation improves local-regional control and survival for selected patients with locally advanced breast cancer treated with neoadjuvant chemotherapy and mastectomy. $J$ Clin Oncol. 2004;22(23):4691-9.

31. Poortmans PM, Collette S, Kirkove C, et al. Internal mammary and medial supraclavicular irradiation in breast cancer. $N$ Engl $J$ Med. 2015; 373:317-27. 
32. Whelan TJ, Olivotto IA, Parulekar WR, et al. Regional nodal irradiation in early-stage breast cancer. $N$ Engl $J$ Med. 2015;373:307-16.

33. Kim KH, Noh JM, Kim YB, et al. Does internal mammary node irradiation affect treatment outcome in clinical stage II-III breast cancer patients receiving neoadjuvant chemotherapy? Breast Cancer Res Treat. 2015;152:589-99.

34. Luo J, Jin K, Chen X, et al. Internal Mammary Node Irradiation (IMNI) improves survival outcome for patients with clinical stage II-III breast cancer after preoperative systemic therapy. Int $J$ Radiat Oncol Biol Phys. 2019;103:895-904.
35. Recht A, Comen EA, Fine RE, et al. Postmastectomy radiotherapy: an American Society of Clinical Oncology, American Society for Radiation Oncology, and Society of Surgical Oncology Focused Guideline Update. J Clin Oncol. 2016;34(36):4431-42. 